\title{
Chronic hepatitis c in PWIDS, treat or not to treat
}

\section{Introduction}

Hepatitis $\mathrm{C}$ virus has been one of the most potential pathogens that have hindered the medical community all over the world. The data reported by $\mathrm{WHO}$ estimated that the prevalence of $\mathrm{HCV}$ infection is $2.2 \%$, and more than one million new cases were reported annually. Furthermore, an estimated $27 \%$ of cirrhosis and $25 \%$ of hepato-cellular carcinomas (HCC) worldwide occur in HCV-infected people. Such infection increases tremendously among the developing countries particularly at those categories that were considered to be at a potential risk of acquiring hepatitis $\mathrm{C}$ virus. ${ }^{1}$ As the world health organization has put a goal of elimination of $\mathrm{HCV}$ and as a global project, it should focus not only on prevention, but also in treating existing patients with a special care for the high transmitter group as prisoners and people who inject drugs. Recently, there was a debate concerning treating those patients or no for the assumption that they do not respond well and the high re-infection rate.

\section{Prevalence}

If we look at the estimated prevalence of HCV among PWIDs worldwide, we can notice that this patient population constitutes the major percentage of the $\mathrm{HCV}$ patient ranging between $40 \%-80 \%$ in 49 countries and reaching more than $80 \%$ in 12 countries. $^{2}$

\section{Efficacy}

Unlike the common concept that PWIDs respond less than general population to anti-viral treatment, retrospective analysis of ION 1,2 and 3 trials of patients on stable substitution replacement therapy (OST) versus non OST participants who were treated with combination of Sofosbuvir with Ledipasvir plus or minus Ribavirin, showed No differences between OST and non-OST participants with overall SVR12 (94\% vs 97\%, p=0.29), adherence to LDV/ SOF $\geq 80 \%$ (94\% vs $96 \%, p=0.33)$ and Proportion with AEs $(89 \%$ vs $80 \%, \mathrm{p}=0.07)$. No cases of HCV re-infection were observed up to SVR24. ${ }^{3}$ Other Phase 2, open-label, single arm study of OMV/ PTV/RTV+DSV for 12 weeks in GT1 adult patients on stable OST with methadone or buprenorphine $(\mathrm{N}=38)$ showed SVR 97\% which is almost similar SVR achieved with general population. ${ }^{4}$ Another phase 3, randomised, double-blind study in PWID on OST with GT1, 4 or 6 using Grazoprevir/Elbasvir for 12weeks showed over all SVR for all genotypes $92 \%{ }^{5}$

\section{Adherence}

In a Phase 3, randomised, double-blind study in PWID on OST with GT 1,4 or 6 using ELB/GRZ for 12 weeks in patients on OSTC-EDGE CO-STAR showed that 99\%(199/201) patients completed 12 weeks of treatment with grazoprevir/Elbasvir, majority of patients (97\%) missed 3 doses or fewer. Around $60 \%$ patients tested positive for illicit drug use during the study. ${ }^{5}$

\section{Re-infection}

Re-infection rates are low among PWIDs ('ever' injectors) with Pooled estimate of HCV re-infection risk for PWIDs 2.4(95\% CI 0.9-6.1) per 100person-years. ${ }^{6}$
Volume 3 Issue 4 - 2017

\author{
Motaz Fathy Saad \\ Department of Internal Medicine, Mubarak Al-Kabeer Hospital, \\ Kuwait
}

Correspondence: Motaz Fathy Saad, Department of Internal Medicine, Mubarak Al-Kabeer Hospital, Jabriya, Hawalli, Kuwait, Email motazmowafy@gmail.com

Received: August 30, 2017| Published: August 31, 2017
i. Re-infection rates among PWIDs may be higher
ii. In communities with high $\mathrm{HCV}$ prevalence ${ }^{7}$
iii. For young PWIDs ${ }^{8}$
iv. For active injection drug users. ${ }^{8}$

In a Meta-analysis of 66 studies in 11,071 patients done to evaluate the Risk of HCV re-infection in low and high risk groups and HIV/ $\mathrm{HCV}$ co-infection showed that in 43 studies evaluating patients with low risk to have recurrence rate per 100 patients per year to be 0.23 , while in 16 studies evaluating those with High risk (PWID/prisoners) have recurrence rate of 2.8 per 100 patient per year while those who are co-infected with $\mathrm{HIV} / \mathrm{HCV}$ were found to have recurrence rate of 4.78 per 100 patient per year in 7 studies. ${ }^{9}$ One other point from the personal experience in dealing with this patient population, treating them and giving them the chance to clear the virus and save their livers can motivate some of them to consider quitting drug injection and make them feel that they are being treated like general population without discrimination.

\section{Conclusion}

Patients with chronic hepatitis $\mathrm{C}$ who are active drug injectors or with history of intravenous drug abuse do respond well to anti-viral therapy with comparable cure rates to general population and similar rate of adherence to treatment with low re-infection rate. As PWIDs constitute most of chronic hepatitis $\mathrm{C}$ patients in many countries worldwide, treatment of this patient population is a priority ${ }^{10}$ to achieve the goal of $\mathrm{HCV}$ eradication and to cut the infection circle. In countries which find difficulty in implementation of needle exchange program due to social issues or any other cause, should adopt treatment as prevention strategy among PWIDs.

\section{Acknowledgements}

None.

\section{Conflict of interest}

Author declares that there is no conflict of interest.

\section{References}

1. Mohamed A Daw, Aghnaya A Dau. Hepatitis C Virus in Arab World: A State of Concern. The Scientific World Journal. 2012;2012:1-12. 
2. Grebely J, Dore GJ. Can hepatitis C virus infection be eradicated in people who inject drugs? Antiviral Res. 2014;104:62-72.

3. Jacobson I. AASLD. Poster \#1945, USA; 2014.

4. Lalezari J, Sullivan JG, Varunok P, et al. Ombitasvir/paritaprevir/r and dasabuvir plus ribavirin in HCV genotype 1-infected patients on methadone or buprenorphine. J Hepatol. 2015;63(2):364-369.

5. Dore G. AASLD. Oral \#49, USA; 2015.

6. Aspinall EJ, Corson S, Doyle JS, et al. Treatment of hepatitis C virus infection among people who are actively injecting drugs: a systematic review and meta-analysis. Clin Infect Dis. 2013;57(suppl 2):s80-s89.
7. Grady BP, Schinkel J, Thomas XV, et al. Hepatitis C virus reinfection following treatment among people who use drugs. Clin Infect Dis. 2013;57(suppl 2):s105-s110.

8. Aitken CK, Lewis J, Tracy SL, et al. High incidence of hepatitis C virus reinfection in a cohort of injecting drug users. Hepatology. 2008;48(6):1746-1752.

9. Hill A. CROI. Abstract \#654, USA; 2015.

10. EASL Recommendations on Treatment of Hepatitis C. Journal of Hepatology. 2016;66(1):153-194. 